\title{
IN SEARCH OF THE ELUSIVE LONG-WAVE FUNDAMENTAL
}

\author{
M. Alpern, B. Bastian* and J. Moeller ${ }^{*}$ \\ Vision Research Laboratory, University of Michigan, Ann Arbor, \\ MI 48109, U.S.A.
}

\begin{abstract}
Action spectra for threshold detection of flicker $(30 \mathrm{~Hz})$ were oblained on 11 deuleranopes under carefully controlled adaptation conditions. Individual differences were large, so that each one of the long-wave fundamentals proposed by different theorists finds reasonable justification in the spectrum measured on at least one deuteranope. Some deuteranopes' spectra are not described by any one of these "fundamentals". To a first approximation at least, trichromats' spectra show the property of linear additivity. One such trichromat's spectrum agreed well with that of a deuteranope with whom he shares a common erythrolabe, and appears to be uninfluenced by his chlorolabe-filled cones.
\end{abstract}

The development of modern color theory since Thomas Young (1807) relies heavily on the view that the vision of congenital dichromats are reduced forms of normal trichromacy. William Rushton (1965) made a fundamental advance in this regard by providing direct evidence that in the retina of deuteranopes the middle-wave foveal cone visual pigment (he named it "chlorolabe") was missing. This excluded an alternative hypothesis (Fick, 1897) that deuteranopes have all three normal cone visual pigments with their dichromacy resulting from some fusion of nerve connections [cf. also Alpern and Wake (1977)]. Two years earlier, Rushton (1963) showed that a long-wave cone visual pigment ("erythrolabe") was missing in protanopes.

These results, confirming a view of dichromacy first emphasized by Koenig and Dieterici (1886), would seem at a single stroke to settle one of the most controversial issues of color theory, namely the nature of the long-wave "fundamental" of normal foveal trichromacy. One had merely to determine on the color triangle representing normal color vision, dichromatic confusion locuses for each variety of congenital dichromacy. They provide, according to Koenig's hypothesis, the nine coefficients in the three linear equations which relate the three experimentally determined normal color matching functions to the absorption spectra (again at the cornea) of the three cone visual pigments upon which these matches must depend (Wyszecki and Stiles, 1967).

But the matter is far from settled. Given Rushton's $(1963,1965)$ results, it is impossible to doubt Koenig's view, but the nature of the absorption spectrum of erythrolabe eludes agreement among contemporary theorists. Using different assumptions about representative results of normal foveal trichromatic color matching and/or of dichromatic confusions, four dif-

\footnotetext{
* Present address: Jules Stein Eye Institute, UCLA Los Angeles, CA 90024, U.S.A.

+Present address: David Sarnoff Research Laboratories, RCA, Princeton, NJ 08540, U.S.A.
}

ferent estimates of this spectrum are found in the literature since Rushton's discovery (Wyszecki and Stiles, 1967; Vos and Walraven, 1971; Smith et al., 1976; Estévez, 1979), with no unequivocal theoretical way of reaching a decision as to their respective validities. In the first part of this paper, an effort is made to choose experimentally from among these possibilities using a new method to measure the spectral sensitivity (at the cornea) of the long-wave-sensitive cones in a population of deuteranopes. The second part describes a preliminary attempt to analyse the information obtained when the same procedure is carried out on a normal trichromat.

The action spectrum to detect flicker of a light alternating ( $100 \%$ depth of modulation, square wave, unit light-dark ratio) at a fixed frequency $(30 \mathrm{~Hz})$ with foveal vision, has such a long history [cf. for example, Ives (1912a), Allen (1923), Heath (1958), Collins (1961), Pokorny and Smith (1972), Bornstein and Marks (1972) and Bailey (1978) for only a representative sample of this literature] readers may be surprised to find still another paper on the subject, much less one professing to describe a "new" method. Unfortunately, a study of this literature reveals that such work was done prior (or oblivious) to the body of research documenting the important effects adaptation of the retinal area under test, and of its surround, can have upon the amount of light required for threshold flicker detection (Lythgoe and Tansley, 1929; Fry and Bartley, 1936; Alpern and Sugiyama, 1961). The method described here was designed to control such variables as carefully as possible. Designating it as "new" reflects the conviction that new facts revealed by the method which in some instances are inconsistent with results from earlier studies are attributable, at least in part, to attention to such details.

\section{Stimulus conditions}

An objectively flickering light viewed against a surround of a different luminance, though it appears subjectively fused with steady fixation, can as an artifact 
appear to flicker when small eye movements momentarily position the test upon a part of the retina adapted to a different level of illuminance. Controlling fixation well enough to obviate such movements during an experimental session several hours long is difficult, if not impossible, for even the most practiced subject. The magnitude of this artifact is the greater, the larger the contrast between center and surround. and is greatest if the test is bright and the surround black. Lythgoe and Tansley (1929) and Fry and Bartley (1936) prove that the best way of avoiding such artifacts is to surround the flickering test stimulus with an annulus whose luminance is precisely the Talbot luminance of the flickering field. For "white" light testing. this presents no special problems, but for monochromatic test lights varied throughout the spectrum in a given experimental session differences in color between test and surround can cause analogous artifacts (Allen, 1923). Zwas (1979) obviated this difficulty by keeping the spectral composition of test and surround always identical; but with this procedure the various points on the action spectrum are obtained under different chromatic as well as luminance adaptation conditions. His action spectra for deuteranopes and protanopes are quite irregular perhaps in part for this reason. The alternative. the one followed here, is to make all measurements with a neutral surround.

\section{METHOD}

"White" light from the $150 \mathrm{~W}$ xenon lamp (approx. color temperature $5200 \mathrm{~K}$ ) filled the annular surround the o.d. of which subtended $3^{\circ}$ of visual angle. The i.d. of this annulus was $1 \%$, contiguous with the border of the central circular disk - a monochromatic test beam from the same source emerging from the exit slit of a grating double monochromator $(2 \mathrm{~nm}$ half bandwidth) in the tristimulus colorimeter described by Alpern et al. (1976). An episcotister, driven by a constant speed motor, interrupted this light $(30 \mathrm{~Hz})$ at the entrance slit of the monochromator. Subjects viewed the center of the disk with foveal vision through a 1-mm $\mathrm{m}^{2}$ artificial pupil.

With a steady test, a dichromat turned the wavelength drum of the monochromator to find his neutral point, i.e. a wavelength in the spectrum metameric with the $5200 \mathrm{~K}$ surround. The motor driving the episcotister was then started and the test neutral wedge adjusted until the light attenuated by it was at the maximum which just allowed the central disc to appear "fused". The surround intensity was now readjusted making the test and surround again identical. Slight adjustments of the latter often upset the former, but by a series of successive approximations the intensities of both test and surround could quickly be found at which the two were metameric with the former just above the c.f.f. The surround was fixed at this value throughout the experimental session; the wavelength of the test was changed in 10-nm steps over the visual spectral range (usually from 400 to $700 \mathrm{~nm}$ ).

A second, and more pernicious, artifact is that the threshold c.f.f. is driven up, or down, as the subject adapts to a light alternating above, or below, the c.f.f. respectively (Alpern and Sugiyama, 1961). This complexity can distort any action spectrum obtained in a single experimental session particularly if, as is usual practice, successive wavelengths are tested in any set routine (such as beginning at the violet extreme and moving systcmatically toward the long-wave end in 10-nm step).

The subject looking about the dimmed experimental room shifted gaze to the test which was set about $0.5 \mathrm{log}$ units dimmer than that estimated to produce c.f.f. and therefore appeared steady when directly fixed. Upon taking up fixation he increased the test intensity until it was first seen to flicker; he immediately changed fixation closing his eyc or looking again around the room. (Alpern and Sugiyama's results show that this routine is less sensitive to "driving" than any other possibility.) The experimenter noted the wedge setting, changed the wavelength of the test and reset its intensity to a level estimated to be about $0.5 \mathrm{log}$ unit dimmer than the c.f.f. for this wavelength; a second measurement was then made following the identical procedure.

The wavelengths were randomly selected aided by a table of random numbers, to obviate systematic adaplation effects. The routine continued until the entire spectrum had been tested. Following a short break a second spectral traverse was undertaken in the same way as the first, test wavelengths again being selected at random. On some occasions still a third repetition followed after a second rest period. It was sometimes found that the two (or three) measurements at a given wavelength did not reasonably concur. Wherever that was so, a concluding set of measurements were undertaken, following a final break, devoted to repeating measurements enough times (but only at these wavelengths) to remove the inconsistencies. Where more than one wavelength were tested, randomization within these constraints continued even for this final set. Action spectra from a single experiment were invariably uneven so (in all but two deuteranopes) this entire testing procedure was repeated on at least three and in some cases on as many as eight additional days each separated from its predecessor by an interval of about 1 week. The results of each day session were averaged; the plotted spectra show the mean \pm 1 SEM at each wavelength, results for each day given equal weight.

The details of apparatus design and calibration have already been described (Alpern et al., 1976). Results of measurements are given on one protanope, 11 deuteranopes and one normal trichromat below.

Dichromats were recruited and the diagnosis established using the routine of this laboratory (Alpern and Wake, 1977). All subjects were white male students at this university, mainly undergraduates, in the second 
PART I-DEUTERANOPES

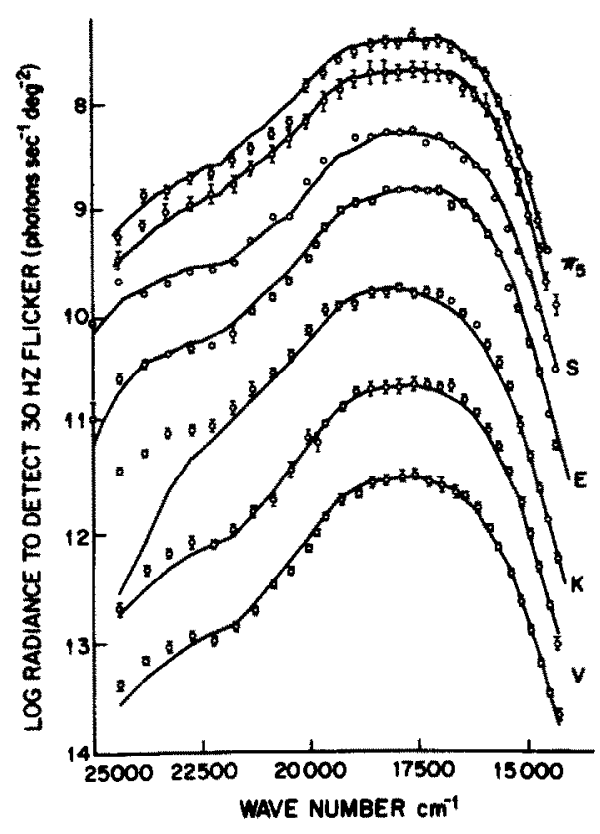

Fig. 1. The action spectrum to detect flicker for each of seven deuteranopes. Plotted as points are the means \pm 1 SEM of the determinations for each subject. The results are reasonably described by one of the five fundamental templates. The optimum fitting template has been slid vertically to minimize the discrepancies as judged by eye and is drawn in the figure as a solid line. The template is identifed in each case at the extreme right: Stiles long-wave cone mechanism field sensitivity $\left[\Pi_{5}(\mu)\right]$, Smith et al. (S), Estévez (E), Vos-Walraven (V) and Koenig (K). Only the fit of the last is unsatisfactory. The ordinate scale is appropriate for the two uppermost spectra; the remaining sets of data have been arbitrarily displaced downward, in succession $0.8 \mathrm{log}$ units with respect to the spectrum immediately above it, to clarify the display.

or third decade of life. Many of them were also included in previous studies of deuteranopes described by Alpern and Wake (1977) and Alpern and Pugh (1977).

The threshold criterion (just to detect flicker at $30 \mathrm{~Hz}$ ) sets the level of retinal illuminance of the surround at about $2.0 \log \mathrm{td}$. This is near the point of maximum slope of the Ferry-Porter curve, hence the region of greatest sensitivity to flicker. The frequency is sufficiently high to insure that short-wave-sensitive cones make no contribution to flicker discrimination (Brindley et al,, 1966; Green, 1969). Since the measurements described in this paper concern only the rod-free fovea, data on protanopes give action spectra of chlorolabe-filled cones, those on deuteranopes, spectra of erythrolabe-filled cones. On the other hand, measurements on normal trichromats provide action spectra to which, it may be assumed, both varieties of cones make a contribution. What that contribution might be is examined in the final part of this paper.

\section{Results}

Results on eleven deuteranopes are shown in Figs 1 and 2, which depict on the ordinate the $\log$ radiance (photons/sec per deg) for threshold detection of $30-\mathrm{Hz}$ ficker plotted as a function of test wave number in $\mathrm{cm}^{-1}$. The ordinate scale is appropriate for the two sets of results at the top of Fig. 1 but each of the others are arbitrarily displaced down by $0.8 \log$ units from the set immediately above it for clarity of the display. (A similar convention governs the organization of Fig. 2 though the ordinate is proper for only the uppermost set and each of the others is successively displaced down $1.0 \log$ units.)

The clearest finding is the remarkable diversity of action spectra specified by the erythrolabes among these 11 deuteranopes. Template curves of the spectra of the long-wave fundamentals suggested by various authorities have been drawn and slid vertically for optimum fit to the results from each deuteranope. The best-fitting template as judged by eye is drawn as a solid line through the experimental points in the first two figures (the top set of results in Fig. 2 is an exception discussed below).

Remarkable in Fig. 1 is that the action spectrum of nearly every recommended fundamental provides a tolerable fit to the erythrolabe action spectrum of at least one deuteranope. The sole exception is the Koenig fundamental tabulated by Wyszecki and Stiles (1967) ( $\mathrm{K}$ in Fig. 1 fit to the third spectrum from the bottom) which is too insensitive for green, blue-green, blue and violet lights compared with the data of the deuteranope whose erythrolabe spectrum is most closely matched by this template.

The present set of results also show that one or the other of the four fundamentals suggested respectively by Wyszecki and Stiles (1967), Vos and Walraven (1971), Smith et al. (1976) and Estévez (1979) (solid lines labelled: $K, V, S$ and $E$ respectively in Figs 1 and 2) are by no means sufficient to provide a satisfactory fit to every erythrolabe action spectrum measured in this sample. For example $\Pi_{5}(\mu)$ the field sensitivity of the long-wave foveal cone mechanism defined by the two-color increment-threshold technique of Stiles $(1939,1949,1953,1959)$ gives a much more satisfactory estimation of the two erythrolabe spectra plotted at the top of Fig. 1 than any of the four recommended fundamentals [These fits of $\Pi_{3}(\mu)$ are given by the solid line drawn through the top two spectra in Fig. 1. The agreement with the uppermost set is only fair but that for the spectrum second from the top illustrates the most satisfactory agreement between template and measurements in the sample.] This may surprise those familiar with this literature. Though the suggestion has been occasionally made [Stiles, 1946; Pugh and Sigel, 1978; Siegel and Pugh, 1980] that the field sensitivity of $\Pi_{5}(\mu)$ provides a valid operational definition of the action spectrum of long-wave-sensitive cones, the conventional wisdom among color author- 
ity, influenced no doubt by Wald's (1964) conviction that $\Pi_{5}$ was much too broad to define erythrolabe in deuteranopes. is that this is not the case.

It is by no means easy, however, to account for the action spectrum of every deuteranope's erythrolabe, even granting flexibility to choose one from among the five template curves examined in Fig. 1. The results in Fig. 2 show the four other examples of deuteranope long-wave cone action spectra. These are not well described by any one of the five. The lowermost set fits the Vos-Walraven template about as well (or as poorly) as the relevant spectrum in Fig. 1 is described by the Koenig fundamental $(\mathrm{K})$. The two immediately above it are also "best fit" by Vos-Walraven but the results are even worse. Finally any one of the five templates applied to the uppermost spectrum provides a quite generally unacceptable fit. [To emphasize this last. the two extreme templates, $\Pi_{5}(\mu)$ (dotted line) and the Koenig fundamental (solid line) is each normalized at its peak to these data.]

Evidently the concept of a "fundamental" action spectrum based on the confusion locus of a single deuteranope, or the average locus of a population of deuteranopes. is inconsistent with the systematic individual differences found in the present study. While these techniques do not exclude the possibility that all individual differences are due to the trivial cause (differences in the losses in the eye media), the "fundamental" spectra themselves as well as the data to which they are most often applied in one theoretical context or another are likewise only capable of measurement at the point they enter the eye. On the other hand, techniques which do allow that distinction to be drawn have already been used by Alpern and Pugh (1977) on a population of deuteranopes including most (but not all) of the present sample. They excluded the hypothesis that all differences in erythrolabe action spectra measured by their methods could be dismissed with this trivial alternative to individual differences in erythrolabe extinction spectra between one deuteranope and the next. The present results are consistent with and indeed offer further evidence in support of, the suggestion of Alpern and Pugh (1977) that there can be no single long-wave "fundamental" for every normal trichromat, for every deuteranope, or for that matter, every tritanope. Rather we suppose a population, (Alpern and Pugh's term was "cluster") of different erythrolabes each differing from the others in the peak wavelength of its extinction spectrum. In the Alpern and Pugh view, a given normal trichromat and a deuteranopic dichromat generally will not both have erythrolabes with identical extinction spectra though this possibility is by no means excluded. But such identity, according to the hypothesis, is not at all certain a priori, it can only be established by experiment: the deuteranope must accept each one of the trichromat's color matches. When this was rigorously tested in the analytical anomaloscope of Baker and Rushton (1963), Alpern and Pugh searched unsuccessfully for any deuteranope with whom exact agreement with one normal trichromat could be reached. In the present sample of 11 deuteranopes, one was found to agree in this way with the color matches of one of three normal trichromats. The comparison of the flicker matches of these two observers, a dichromat and a trichromat both of whose longwave cones have erythrolabe with identical absorption spectra is useful for analysing the spectra obtained by the application of the present method to the foveas of normal trichromats. This is considered next.

\section{PART II NORMAL TRICHROMATS}

Rushton's (1963. 1965) proof that each variety of red-green dichromat lacks one of two foveal cone pigments maximally absorbing in the red-green spectral range, gives strong reason to suppose that the procedure described in this paper measures the action spectra of a single species of cone when carried out on the foveas of protanopes and deuteranopes. But what does it measure on the normal fovea? How do those two species of cones of the normal retina capable of following $30-\mathrm{Hz}$ flicker, pool their respective signals

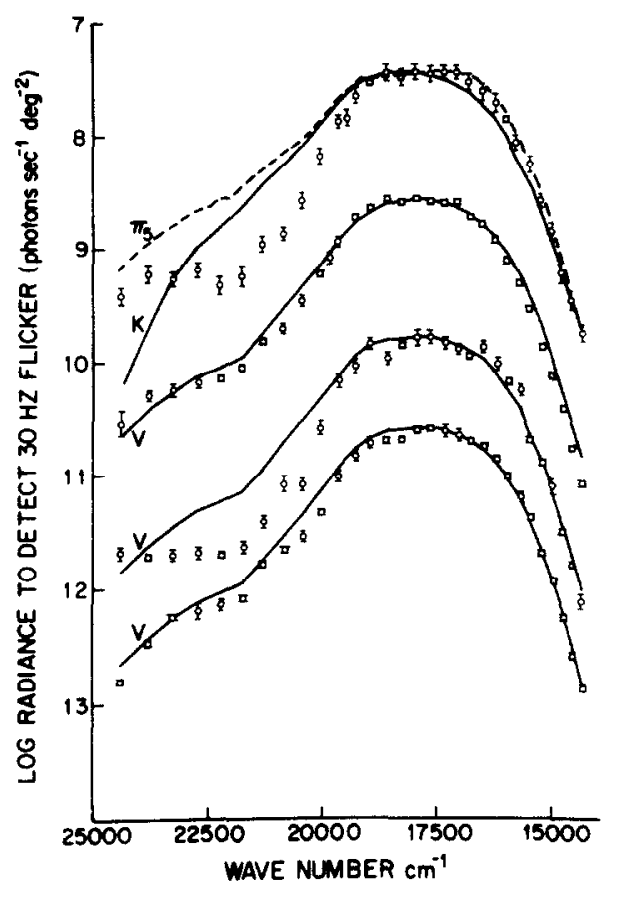

Fig. 2. Action spectra to detect flicker of four additional deuteranopes as in Fig. 1. For these dichromats not one of the five templates adequately describes the data. The solid curve drawn through the three lower sets is the Vos-Walraven ( $V$ ) fundamental which in these cases (however imperfectly the fit is) does a better job that any of the others. The topmost curve show the most extreme misfit of data with templates found. The two curves $\left[\Pi_{5}(\mu)\right]$, dashed line and $(K)$ solid line, show the template extremes. Ordinate scale is correct for this set of results; the others have each been arbitrarily displaced downward $1.0 \mathrm{log}$ units in succession with respect to the spectrum immediately above it. to clarify the display. 
to yield threshold flicker? The complexity of the human visual system would on the surface appear to preclude any psychophysical experimental result leading to a strong inference which might answer this question. However, it was proved by Enroth-Cugell and Robson (1966), for the cat at least, that some signals must pool at the retinal ganglion cell level maintaining a linear relation between signal size and rate of photon absorption. While it is true, of course, that pooling of this kind can almost never be documented between different species of cones in the responses measured psychophysically with human eyes. if they did apply in this case, it would enormously increase the possibility of achieving a relatively simple analysis of the problem. Encouraged by the experiments of Ives (1912b) on flicker photometry, if not by c.f.f. results of Trick and Guth (1981) (who tested flicker at lower frequencies against dark surround) the following experiment was undertaken to examine the outside chance pooling might be linear. It is modelled after the experiments of Boynton et al. (1964) who raised a similar question with regard to the longwave- and short-wave-sensitive foveal cones in the two-color increment-threshold paradigm of Stiles (1939).

\section{Method}

A normal trichromat served as subject. The apparatus used all three channels of the Maxwellian view apparatus of Alpern and Zwas (1979). Two of these provided 1.2 dia circular test fields spatially superimposed which could be flickered $(30 \mathrm{~Hz}$, square wave, $100 \%$ modulation depth, unit light-dark ratio) in perfect phase. Beam (1) contained a Baird-Atomic interference filter transmitting $460 \pm 10 \mathrm{~nm}$, the other beam (2) contained a similar filter transmitting $650 \pm 10 \mathrm{~nm}$. These two beams were orthogonally polarized and mixed together. After mixing they passed through a rotating analyser and a calibrated counter balanced "neutral" common wedge. The o.d. of this field was contiguous with the i.d. of the concentric circular annular surround-a steady "white" light (color temperature $5200 \mathrm{~K}$ ), $8^{\circ}$ o.d., provided by the third channel of the apparatus. All three fields were seen in sharp focus in Maxwellian view: the largest dimension of the filament image in the subject's pupil from the surround field was $0.25 \mathrm{~mm}$, those from the two tests were $1.0 \mathrm{~mm}$. These images were superimposed, centered in the subject's pupil. To equate the luminance of the neutral surround the $650-\mathrm{nm}$ test was occluded by rotation of the analyser for maximum intensity of transmission of the $460-\mathrm{nm}$ test, and the wedge common to the two test beams was set so that the test just "fused". A counter balanced wedge in the surround beam was adjusted to match the Talbot luminance of the 460-nm test ignoring hue differences. The surround luminance for any given session was fixed by the average of five settings. Five successive flicker thresholds were then made of the 460-nm test with the common test wedge, follow- ing the precautions regarding the adaptation and fixation previously described in this paper. This wedge was fixed in the average position and the analyser rotated through $90^{\circ}$ occluding the $460-\mathrm{nm}$ test and exposing the maximum intensity of $650-\mathrm{nm}$ test. Five settings of threshold c.f.f. were made by adjusting the transmission of a third counter-balanced wedge in the red test field. This wedge was fixed at the average position throughout the remainder of the session.

Having fixed the intensity of the surround and the thresholds for the $460-\mathrm{nm}$ test and 650 -nm test each by itself alone, the additivity of the two was tested in different combinations by setting the analyser in 8-10 intermediate positions. For each setting, the subject adjusted the common test wedge for just-perceptible flicker following the adaptation and fixation routine described above. The analyser positions for each successive setting were randomized, as in the previous experiments, and the experiment continued until five settings had been made for each mixture combination.

\section{Results}

Results were analysed utilizing the "S units" of Boynton et al. (1964): let $R_{10}, R_{20}$ designate threshold radiance to detect flicker with the $460-\mathrm{nm}$ and $650-\mathrm{nm}, 30-\mathrm{Hz}$ test alone, respectively, and $R_{1 M}, R_{2 M}$ those radiances at threshold of the mixture; define new units (called " $S$ units") in terms of which the stimulus values become $S_{1}$ and $S_{2}$ such that

$$
\begin{aligned}
& S_{1}=R_{1 M} / R_{10} . \\
& S_{2}=R_{2 M} / R_{20} .
\end{aligned}
$$

The experiment tests the linearity of the response by determining for each mixture how closely $S_{1}+S_{2}=1.0$. The results of this experiment carried out in two different experimental sessions are given by triangles (for $460 \mathrm{~nm}$, i.e. $S_{1}$ ) and squares (for $650 \mathrm{~nm}$, $S_{2}$ ) respectively in the upper part of Fig. 3 .

In this figure the ordinates show $\log S_{1}$ (or $\log S_{2}$ ) while the abscissa gives different values of $\log r=\log$ $\left(S_{2} / S_{1}\right)$. The solid curves are the expectations if the two lights added linearly at threshold, i.e. $S_{1}+S_{2}=1.0$. Open and filled symbols show results obtained on different days.

These results in Fig. 3 concur very nearly with additivity, though there are small but perhaps systematic deviations in the direction of subadditivity. These discrepancies are never very large; on only two occasions did discrepancies equal or exceed $0.1 \log _{10}$ units and neither was repeated in both runs. This is easier to appreciate by study of the circles at the bottom of Fig. 3 plotted as $\log \left(S_{1}+S_{2}\right)$ on the ordinate scale. The horizontal line is the expectation of perfect additivity; in general, the results fall slightly above this line, though the average deviation $0.04 \pm 0.011 \log _{10}$ units is of the same order of magnitude as the precision of measu ing the radiance of the light. In general, these experiments imply that threshold flicker 


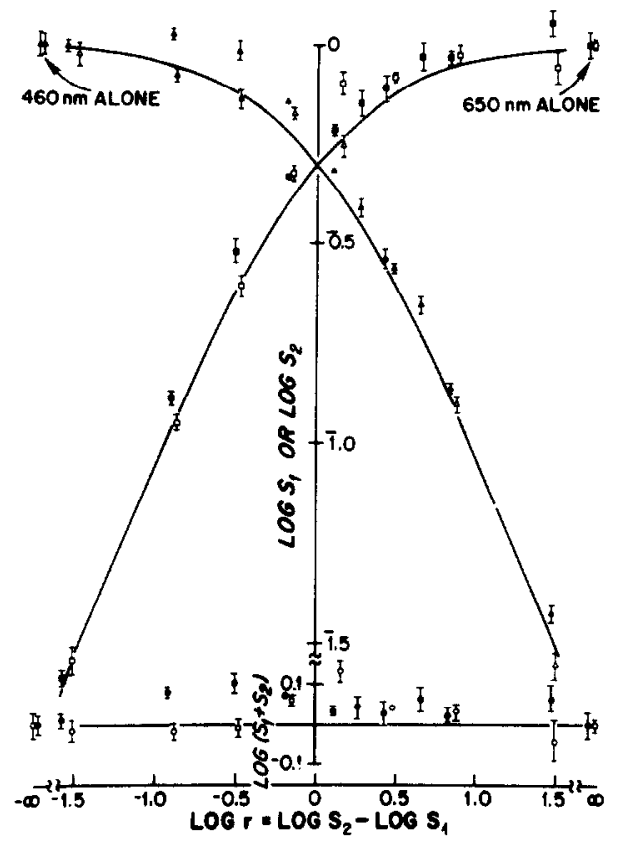

Fig. 3. Additivity of violet $\left(460 \mathrm{~nm}, S_{1}\right)$ and red $(650 \mathrm{~nm}$, $S_{2}$ ) lights for threshold detection of flicker in $100 \%$ modulation square waves, unit light-dark ratio, $30 \mathrm{hz}$. Above: $\log S_{1}$ (triangles), $\log S_{2}$ (as squares) are plotted as a function of $\log r=\log \left(S_{2} / S_{1}\right)$. The smooth curves illustrate the expectation of perfect additivity. Below: the ordinate scale gives $\log \left(S_{1}+S_{2}\right)$, (circles) shown as a function of the same abscissa. Perfect additivity in this case is defined by a horizontal straight line through 0 . Plotted points are the means \pm 1 SEM of results from a single experimental session; open and closed symbols were obtained on different days.

detection under the tested condition are, at least to a first approximation, linear.

This suggests that further analysis of how the signals from long- and medium-wave-sensitive cones are pooled together to yield the action spectrum for threshold c.f.f. is possible. As a preliminary step, the action spectra to detect flicker c.f.f. of normal trichromats were compared with those of protanopes and deuteranopes. This approach has been followed before notably by Heath (1958), Collins (1960) and Pokorny and Smith (1972) all of whom inferred that deuteranopes were abnormally sensitive to the flicker of red-green spectral lights. That inference came from measurements obtained with a dark surround and taken without regard to the influence adapting to a flickering light may have on detecting c.f.f. (Alpern and Sugiyama, 1960). It seemed useful to re-examine the phenomenon under the more carefully controlled testing conditions described above. In doing so, the effort was also made to identify dichromats and normal trichromats whose retinas contained the identical cone visual pigments in the red-green spectral range. Such observers will make identical matches in the Rushton analytical anomaloscope (Baker and Rushton, 1963) when the trichromat confronts the dichro- mat matches with the red-green primaries of the instrument set in the latter's "mode" (cf. Mitchell and Rushton, 1971; Alpern and Pugh, 1977; Alpern and Moeller, 1977). This criterion for comparing the sensitivity of dichromats with normal trichromats is more rigorous than any previously employed in such studies, but it is looser than one might wish because matches in Rushton's anomaloscope are constrained to the red-green part of the spectrum where most trichromats succeed in matching with only two primaries. Even with this limitation, extensive search failed to uncover any normal trichromat for whom it was possible to find both a matching protanope and a matching deuteranope. One trichromat was identified who came very close to fulfilling these requirements with a particular protanope-deuteranope pair. This trichromat agreed precisely with the deuteranope, but did so in only one of three confrontations with the protanope in the analytical anomaloscope. [In the other two protanope confrontations, very small differences (never exceeding $0.03 \mathrm{log}$ units) were found which were nevertheless statistically significant.] Although this agreement was consequently imperfect, as a first step in the analysis of trichromatic flicker we have compared the action spectra of these three observers. The results are so surprising that similar comparison of such data on other normal trichromats would appear to be an important line of study still to be completed.

\section{Method}

The method has already been described. The retinal illuminance produced by the steady neutral surround for the trichromat was fixed at the average value established by the protanope with the method outlined above. The deuteranope's setting of the intensity of the neutral surround was very nearly identical (within $0.076 \log _{10}$ units on the average). Consequently, sets of deuteranope spectra from three experimental sessions in which measurements were made under the usual dichromat's settings for the surround were compared with his spectra from three experiments in which the average of this protanope's surround setting were used. Since the differences were about the same as those measured under comparable conditions with the usual surround settings (and showed no obvious wavelength dependent trend) both sets were pooled and the means plotted as the circles in Fig. 4.

The action spectra required just to detect flicker are shown in Fig. 4 for the normal trichromat as rectangles connected by the solid line, as triangles for the protanope and (as noted) circles for the deuteranope at each wave number $\left(\mathrm{cm}^{-1}\right)$. The figure shows one standard error on either side of the mean value for each observer. It is noteworthy that the ordinate is in absolute radiance units (log photons/sec per $\mathrm{deg}$ ) and that the three sets of spectra are not arbitrarily shifted. There is rather remarkable agreement of the spectra measured on the deuteranope and the normal trichromat; no agreement at all between the latter 


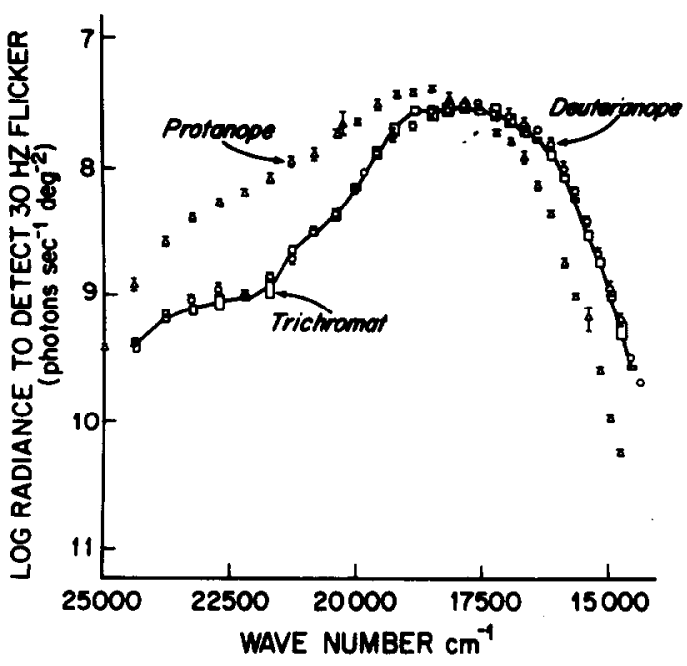

Fig. 4. Action spectra for threshold flicker detection of a protanope (triangles) and a deuteranope (circles); the rectangles connected by a solid line aimed at their centers define 1 SEM on either side of the mean of the settings for each wavelength of the similar action spectrum for that particular normal trichromat whose matches in the analytical anomaloscope agreed everywhere in the red-green part of the spectrum with those of this protanope when the anomaloscopes primaries were equated at their respective maxima according to his protanopic "mode" (or very nearly so) and with those of this deuteranope when the primaries were equated in his deuteranopic "mode". All three curves are properly positioned on the graph without any arbitrary vertical (or horizontal) shifting.

and the protanope's curve. While agreement between the deuteranope's and the trichromat's spectra in Fig. 4 is not perfect (it can be improved slightly by shifting the latter up $0.08 \log _{10}$ units, as if a wavelength-independent criterion differences existed in the measurements of the two observers) it is close enough to suggest the possibility that this action spectrum measured in the normal trichromat's fovea is determined by his erythrolabe-filled cones alone.

\section{DISCUSSION}

If, under the present procedures, erythrolabe-filled cones alone determine the action spectrum of threshold flicker, then it would not be at all surprising that this technique follows the principle of linear additivity as suggested to at least a first approximation by the results in Fig. 3. But how would it be possible to measure any action spectrum at all in the foveal cones of protanopes, which many years ago Rushton (1963) proved contain no erythrolabe? Clearly, whatever the property might be of the normal fovea which causes the action spectrum to be determined by erythrolabe alone, it is a property of cones (or of their wiring) and not of the pigments contained in their outer segments. That the method measures any action spectrum at all in the fovea of a protanope, suggests that in the protanope fovea the cones which normally contain erythrolabe are not absent but filled with chlorolabe (the protanope's only red-green pigment).
It is a curious fact that it was the expectation that an analogous state of affairs might be true for deuteranopes (i.e. that the chlorolabe-filled cones of the normal, in the deuteranope fovea contained erythrolabe) which led Heath (1958) to discover the super-normal deuteranope c.f.f. threshold in the red-green spectral range. No such super-normal threshold is conspicuous in the present data but this by no means excludes the hypothesis that cones which in the normal retina contain chlorolabe, in the deuteranope fovea contain erythrolabe. Indeed, this present result is quite consistent with that hypothesis.

What the network wiring which leads to the result in Fig. 4 may be, can only be a source for speculation. Before exerting too much effort in that direction it is important to know how general these results are for every normal trichromat. This is not an easy question to answer in a compelling way because of the great difficulty of finding for each normal trichromat under study, the precise protanope and deuteranope who will accept his matches (and vice versa) in the rigorous testing of the analytical anomaloscope. Though so far we have only partly succeeded in this task with a single normal trichromat, continuous systematic study should uncover others. The point is in portant not only because of its potential ability to elucidate how the eye processes temporal information but also for the practical advantages it may offer as a useful operational definition of the action spectrum of human long-wave-sensitive cones. Until now, the best such definition would appear to be the field sensitivity action spectrum $\Pi_{S}(\mu)$ of W. S. Stiles (Pugh and Sigel, 1978; Sigel and Pugh, 1980) which defines a rather tedious and time-consuming operation. Comparison of these two operational definitions of the action spectrum of long-wave-sensitive cones on a population of normal trichromats would provide a further validation of the action spectrum (but not the absolute sensitivity) of the threshold flicker procedures outlined here, hence a further way of documenting whether the agreement of the deuteranope and normal trichromat spectra in Fig. 4 is fortuitous or fundamental.

Whatever the outcome of such experiments, the present results emphasize the futility of the search for a single long-wave fundamental which could bc of theoretical relevance to the color vision of every normal trichromat. Rather, these results mean that the application of an absorption spectrum of the longwave-sensitive cones in the theoretical description of the color vision of any normal trichromat, should more realistically depend upon an empirical determination of that spectrum on that same trichromat's fovea. Whether this can best he achieved by methods outlined in this paper, by the Stiles $\Pi_{5}(\mu)$ field sensitivity or by some other as yet undefined operation remains to be decided. But that if theory and color measurements are to be compellingly compared, some such empirical data will be of enormous value, if not a necessity, there can be no doubt. 
Acknowledgement-Assisted by a grant EY-00197-23 from the National Eye Institute to M. Alpern.

\section{REFERENCES}

Allen F. (1923) On refex visual sensations and color contrast. J. opt. Soc. Am. 7, 913-942.

Alpern M., Bastian B., Pugh E. N. and Gras W. (1976) Altered ocular pigments, photostable and labile: two causes of deuteranomalous trichromacy. Mod. Prob. Ophthal. 17, 273-291.

Alpern M. and Moeller J. (1977) The red and green cone visual pigments of deuteranomalous trichromacy. $J$. Physiol. 266, 647-675.

Alpern M. and Pugh E. N., Jr (1977) Variation in the action spectrum of erythrolabe among deuteranopes. $J$. Physiol. 266, 613-646.

Alpern M. and Sugiyama S. (1961) Photic driving of the critical flicker frequency. J. opt. Soc. Am. 51, 1379-1385.

Alpern M. and Wake T. (1977) Cone pigments in human deutan colour vision defects. J. Physiol. 266, 595-612.

Alpern M. and $Z$ was $F$. (1979) The wavelength variation of the directional sensitivity of the Stiles $\Pi_{1}(\mu)$. Vision Res. 19, $1077-1087$.

Bailey J. E. (1978) Flicker fusion thresholds of red-green dichromats. Mod. Probl. Ophthal. 19, 201-205.

Baker H. D. and Rushton W. A. H. (1963) An analytical anomaloscope. J. Physiol. 168, 31-33P.

Bornstein M. H. and Marks L. E. (1972) Photopic luminosity measured by the method of critical frequency. Vision Res. 12, 2023-2033.

Boynton R. M., Ikeda M. and Stiles W. S. (1964) Interactions among chromatic mechanisms as inferred from positive and negative increment thresholds. Vision Res. 4, 87-117.

Brindley G. S., Du Croz J. and Rushton W. A. H. (1966) The flicker fusion frequency of the blue-sensitive mechanism of colour vision. J. Physiol. 183, 497-500.

Collins W. E. (1961) Luminosity functions of normal, deuteranomalous, and deuteranopic subjects as determined by absolute threshold and CFF measurements. $J$. opt. Soc. Am. 51, 202-206.

Enroth-Cugell C. and Robson J. G. (1966) The contrast sensitivity of retinal ganglion cells of the cat. J. Physiol. 187, 517-552.

Estevez O. (1979) On the fundamental data-base of normal and dichromatic color vision. Doctor's thesis, Amsterdam University.

Fick A. (1897) Die Lehre von der Lichtempfindung. In Handbuch der Physiologie (Edited by Hermann L.), Vol. 3, pp. 139-234. Vogel, Leipzig.

Fry G. A. and Bartley S. H. (1936) The effect of steady stimulation of one part of the retina upon the critical flicker frequency in another. J. exp. Psychol. 19, 351-356.

Green D. G. (1969) Sinusoidal flicker characteristics of the color-sensitive mechanisms of the eye. Vision Res. 9. $591-601$.

Heath G. G. (1958) Luminosity curves of normal and dichromatic observers. Science 128, 775-776.
Ives H. E. (1912a) Studies in the photometry of lights of different colours. II. Spectral luminosity curves by the method of critical frequency. Phil. Mag. 24, 352-370.

Ives H. E. (1912b) Studies in photometry of lights of different colors. IV. The addition of luminosities of different colors. Phil. Mag. 24, 845-853.

Koenig A. and Dieterici C. (1886) Die Grundempfindungen und ihre Intensitaetsvertheilung im Spectrum. S. ber. Akad. Wiss. Berlin 805-829.

Lythgoe R. J. and Tansley K. (1929) The adaptation of the eye: its relation to the critical frequency of flicker. Report of the Committee upon the Physiology of Vision, No. 5. Spec. Rep. med. Res. Counc. Ser. 134, 172.

Mitchell D. E. and Rushton W. A. H. (1971) The red/green pigements of normal vision. Vision Res. 11, 1045-1056.

Pokorny J. and Smith V. C. (1972) Luminosity and CFF in deuteranopes and protanopes. J. opt. Soc. Am. 62, 111-117.

Pugh E. N., Jr. and Sigel C. (1978) Evaluation of the candidacy for the $\Pi$ mechanisms of Stiles for color-matching fundamentals. Vision Res. 18, 317-330.

Rushton W. A. H. (1963) A cone pigment in the protanope. J. Physiol. 168, 345-359.

Rushton W. A. H. (1965) A foveal pigment in the deuteranope. J. Physiol. 176, 24-37.

Sigel C. and Pugh E. N.. Jr (1980) Stiles's $\Pi_{5}$ color mechanism: tests of field displacement and field additivity properties. J. opt. Soc. Am. 70, 71-81.

Smith V. C.. Pokorny J. and Starr S. J. (1976) Variability of color mixture data-I. Interobserver variability in the unit co-ordinate. Vision Res. 16, 1087-1094.

Stiles W. S. (1939) The directional sensitivity of the retina and the spectral sensitivities of the rods and cones. Proc. R. Soc. B. 127, 64-105.

Stiles W. S. (1946) A modified Helmholtz line element in brightness colour space. Proc. phys. Soc., Lond. 58, 41-65.

Stiles W. S. (1949) Increment thresholds and the mechanisms of colour vision. Doc. Ophthalm. 3, 138-163.

Stiles W. S. (1953) Further studies of visual mechanisms by the two-colour threshold technique. Colloq. Probl. Opt. Vis. (U.I.P.A.P. Madrid), Vol. 1, 65-103.

Stiles W. S. (1959) Color vision: The approach through increment-threshold sensitivity. Proc. natn. Acad. Sci., U.S.A. 45, 100-114.

Trick G. L. and Guth S. L. (1980) Heterochromatic additivity and the CFF response. Am. J. Opt. Physiol. Opt. 57, 791-794.

Vos J. J. and Walraven P. L. (1971) On the derivation of the foveal receptor primaries. Vision Res. 11, 799-818.

Wald G. (1964) The receptors of human color vision. Science 145, 1007-1016.

Wyszecki G. and Stiles W. S. (1967) Color-Science. Concepts and Methods, Quantitative Data and Formulas. John Wiley, New York.

Young T. (1807) Catalogue - physical optics, confusion of colours. A Course of Lectures on Natural Philosophy and the Mechanical Arts, Vol. 2, pp. 315-316. Joseph Johnson. London.

Zwas F. (1979) The wavelength variation of the directional sensitivity of the long and medium wave sensitive foveal cones of red-green dichromats. Vision Res. 19, $1067-1076$. 